\title{
Richard Mollica, Md, Mar. Texto de salud mental global: trauma y recuperación
}

Richard Mollica, Md, Mar. Textbook of global mental health: trauma and recovery

An Fac med. 2014;75(3):273-4 / doi: http://dx.doi.org/10.15381/anales.v75i3.9786

Alberto Perales-Cabrera

'Instituto de Ética en Salud, Facultad de Medicina, Universidad Nacional Mayor de San Marcos, Lima, Perú.

\section{SEÑOR EDITOR:}

La presente es para comunicar sobre el libro de Richard Mollica. Md, mar. Texto de Salud Mental Global: Trauma y Recuperación. Guía para la atención clínica y de campo de víctimas de trauma en el mundo entero (Textbook of global mental health: trauma and recovery. A companion guide for field and clinical care of traumatized people worldwide). Editado por Harvard Trauma Refugee Program, USA, 2011, su versión electrónica puede ser obtenida por US\$60.00 en: http:// www.lulu.com/shop/richard-f-mollica/ textbook-of-global-mental-healthtrauma-and-recovery-a-companionguide-for-field-and-clinical-care-oftraumatized-people-worldwide/ebook/ product-16599951.html.

Con una carátula en la que, a pesar del enorme sufrimiento de su población causado por el devastador terremoto que azotara Haití en el 2010, se observa una joven mujer haitiana con su típica vestimenta blanca, bailando y entregándose al placer del movimiento rítmico, buscando aliviar penas y amarguras contra el destino. Richard Mollica, Profesor de Psiquiatría de la Facultad de Medicina de la Universidad de Harvard, Director del Programa de Recuperación de Pacientes Traumatizados y autoridad mundial en el campo de la investigación en violencia, edita su última obra con el título que encabeza el presente comentario. La anterior, sobre el tema - "Healing Invisible Wounds: Paths to Hope and Recovery in a Violent World", se mantiene como fuente de consulta.

Confiesa el autor que el libro que reseñamos nace de la histórica reunión mundial de Ministros de Salud realizada en Roma, en el 2004 (denominada "Project 1 billion for the number of people affected by violence"), para establecer y difundir el Primer Plan de Acción de Salud Mental Global.

Además de su valioso contenido, el Texto constituye documentada guía para los profesionales de salud y personal voluntario que intentan la recuperación de seres humanos traumatizados por la violencia, así como para los funcionarios responsables de formular políticas de atención para estos casos.

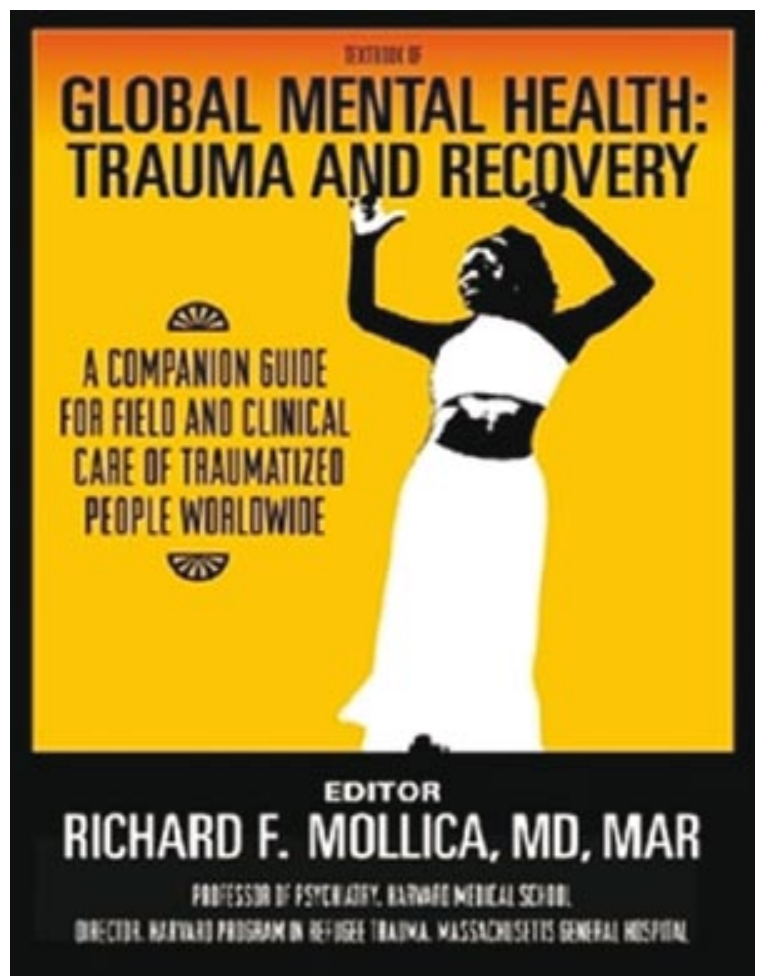


Sus capítulos, organizados en torno a las ocho dimensiones del citado Plan, traducen la colaboración de 41 reconocidos expertos, cubriendo aspectos teóricos, económicos, de derechos humanos, religioso-espirituales, y de ética e investigación, necesarios para la organización de políticas de atención de víctimas de la violencia humana y de desastres naturales.

El aporte de Richard Mollica no solo refleja la calidad de su experiencia clínica cimentada en el arduo trabajo de ayudar a este tipo de pacientes sino, también, un gran valor humanista y solidario con los seres afectos. Tan sa- brosa mezcla solo es posible cuando se han dedicado años de vida profesional a estos menesteres, angustiándose ante el injusto destrozo personal y familiar de tanta gente y estremeciéndose con impotente rabia al apreciar la inoperancia -sino el desdén- de las políticas gubernamentales de ayuda. Se entiende por ello que uno de sus párrafos exprese: "No hay medicación o medidas de seguridad preventivas que puedan proteger al médico y al paciente del abrumador distrés emocional que producen las tragedias humanas de gran escala. Se requiere investigación para implementar las políticas -usualmente caóticas, desorganizadas y culturalmente inadecuadas".
Siendo el Perú un país que ha sufrido y sufre los estragos de la violencia en sus múltiples manifestaciones, felicitamos la aparición de la presente obra, cuya lectura deviene en tarea obligatoria para los profesionales de salud mental y de todas las disciplinas conexas.

Correspondencia:

Dr. Alberto Perales-Cabrera

Instituto de Ética en Salud, Facultad de Medicina, Universidad Nacional Mayor de San Marcos, Lima, Perú

Correo electrónico: perales.alberto@gmail.com 